\title{
El derecho a no ser pobre: la lucha contra la pobreza en España en el marco
} de la estrategia Europa 202024

\author{
José A. Estévez Araujo \\ Universidad de Barcelona \\ jaesaraujo@telefonica.net \\ Joan Ramos Toledano \\ Universidad de Barcelona
}

Fecha de recibido: octubre 2016 / Fecha de aprobación: febrero 2017

\section{Resumen}

El presente trabajo trata de analizar las políticas contra la pobreza aplicadas por España en el marco de la estrategia Europa 2020. Estas políticas se han llevado a cabo mediante mecanismos de gobernanza -en concreto, el denominado experimentalismo democrático-, cuya efectividad y legitimidad están en entredicho. Para ello se analizará, de un lado, el Semestre Europeo, que coordina estas políticas a nivel de la UE. Del otro, distintos documentos españoles que se enmarcan en este proceso y que permiten evaluar los niveles de legitimidad democrática de este mecanismo.

\section{Palabras Clave}

Experimentalismo democrático / gobernanza / Semestre Europeo / legitimidad / pobreza

\footnotetext{
${ }^{24}$ Este trabajo es fruto de las investigaciones realizadas hasta el momento en relación a un proyecto titulado "La legitimidad de la gobernanza en tiempos de crisis: análisis del funcionamiento del Método Abierto de Coordinación (MAC) en España, Portugal e Italia de 2010 a 2015". Cuenta con una subvención del Ministerio de Economía y Competitividad (DER2014-53891-P). El IP es uno de los coautores de esta ponencia: José Antonio Estévez Araujo. El equipo de trabajo está integrado por los miembros del Grupo de Investigación de Filosofía del Derecho de la Universitat de Barcelona. El GI está formado por el otro coautor de esta ponencia, Joan Ramos Toledano y también por José Luis Gordillo, Antonio Madrid, Antonio Giménez y Xavier Pedrol, todos ellos Profesores de Filosofía del Derecho de la UB y por los profesores Giovanni Messina y Fabio Ciaramelli, de la Universidad de Catania y de la de Nápoles respectivamente. La Profesora de Sociología de la UB, Trinidad Bretones, también forma parte del equipo de investigación. Colaboran en el proyecto Julia Núñez de la Rosa, doctora en Humanidades por la Universitat Pompeu i Fabra y Marco Antonio Núñez, doctorando del programa "Ciudadanía y Derechos Humanos" de la UB. A su vez, el proyecto se inscribe en un ciclo de investigación del Grupo. Este ciclo se inició en los años noventa. Nuestro objetivo ha sido comprender las transformaciones provocadas por la globalización neoliberal y su impacto en los campos político y jurídico. El ciclo comprende varios proyectos, los dos últimos dirigidos por José A. Estévez Araujo y todos los anteriores por Juan Ramón Capella. La última publicación colectiva del grupo ha sido el libro La democracia en bancarrota (Estévez Araujo \& Messina, 2015).
} 


\section{Abstract}

This paper attempts to analyze policies against poverty undertaken by Spain within the framework of the Europe 2020 strategy. These policies have been applied through mechanisms of governance mechanisms - specifically, the so-called democratic experimentalism- whose effectiveness and legitimacy is at least questionable. To this end, this paper tries to analyze, on one side, the European Semester, which coordinates these policies at an EU level. On the other, various Spanish documents that are part of this process and therefore allow us assess the levels of democratic effectiveness of this mechanism.

\section{Key words}

Democratic experimentalism / governance / European Semester / legitimacy / poverty

\section{Tabla de contenido}

I. Introducción 2. Del experimentalismo democrático al Método Abierto de Coordinación 2.I. El experimentalismo democrático 2.2. Legitimidad 2.3. El Método Abierto de Coordinación 3. El Semestre Europeo y los mecanismos de coordinación de las políticas contra la pobreza 3.l. ¿Qué es el Semestre Europeo (SE)? 3.2. Contextualización de la coordinación de las políticas sociales en el marco del SE 3.3. El Comité de Protección Social 3.4. Legitimidad: democracia y racionalidad 4. Los Programas e Informes Nacionales del Reino de España 5. Eficacia 6. Conclusiones

\section{Introducción}

El objetivo de este texto es el análisis de las políticas contra la pobreza llevadas a cabo en España dentro del marco de la estrategia Europa 2020. Dicho estudio se inscribe dentro de un largo proceso previo de investigación sobre la gobernanza. El tema de la gobernanza ha sido un campo casi exclusivo de politólogos y científicos de la administración. Sin embargo, es del mayor interés para juristas y filósofos del derecho. Hoy en día la mayor parte de la producción y aplicación del Derecho, por ejemplo, en el ámbito de la Unión Europea, se realiza mediante mecanismos de gobernanza. Los procedimientos de la gobernanza compiten con los representativos. Pretenden, además, tener sus propias fuentes de legitimidad. Se presentan como un complemento o incluso como un sustitutivo de la democracia representativa. Sin embargo no han sido objeto de atención de forma sistemática por parte de los filósofos del derecho en España salvo contadas excepciones, como es el caso de Pedro Mercado (Mercado, 2012). ${ }^{25}$

Aquí no hablaremos de la gobernanza en general. Se tratará de una de sus corrientes: el 'experimentalismo democrático'. En particular se hará un estudio de caso sobre el traspaso de esa forma de gobernanza desde Estados Unidos a la Unión Europea (UE) ${ }^{26}$.

\footnotetext{
${ }^{25}$ El italiano Giovanni Messina, miembro de nuestro grupo de investigación ha publicado diversos trabajos sobre la materia. (Messina, 2012)(Messina \& Quiroz Vitale, 2015) V.t. (Estévez Araujo, 2015).

${ }^{26}$ Al final del documento, después de la bibliografía el lector puede encontrar un glosario de las siglas
} 
Nuestro trabajo se centrará fundamentalmente en la evaluación de la legitimidad de los procedimientos de toma de decisiones. Aunque los criterios de legitimidad que reclama el experimentalismo democrático son cuestionables en sí mismos, en este trabajo nuestro objetivo será determinar si realmente se cumplen en la práctica en el estudio de caso que se presenta.

Para abordar esta cuestión, no es suficiente tener en cuenta la constitución estatal. Los estados de la UE, y entre ellos España, están insertos en un sistema jurídico-político 'multinivel' (Poiares Maduro, 2003). No tienen autonomía para determinar sus políticas sociales. Esto era ya cierto en el momento de la entrada de España en la Comunidad Económica Europea y algunos lo señalamos entonces. La pérdida de poder de decisión se acentuó con la creación del euro. La moneda única incrementó la sumisión de los estados a los órganos de la Unión Europea, especialmente el Banco Central Europeo (BCE). En la actualidad, la pérdida de autonomía se ha hecho patente incluso para el gran público. Las medidas de austeridad implantadas como respuesta a la crisis e impulsadas fundamentalmente por Alemania están teniendo consecuencias muy graves para el desarrollo de las políticas sociales ${ }^{27}$.

\section{Del experimentalismo democrático al Método Abierto de Coordinación}

Existen muy diversas corrientes de análisis de la gobernanza e, incluso, dos generaciones diferentes de estudios sobre ese fenómeno ${ }^{28}$. Sin embargo, todas tienen un elemento en común: la participación directa de los stakeholders (interesados) en los procesos de toma de decisiones.

El término 'stakeholders' proviene del ámbito empresarial. Surgió cuando empezó a plantearse la cuestión de la Responsabilidad Social Corporativa (RSC). Hasta entonces se había sostenido que las empresas sólo tenían la responsabilidad de proporcionar beneficios a sus accionistas (shareholders). Frente a esa afirmación, los defensores de la RSC sustentaron que las empresas eran responsables respecto de todas las personas a quienes afectaba su actividad (clientes, vecinos, trabajadores, proveedores, consumidores, etc.). Para referirse a éstos se utilizó el término 'stakeholders', que luego se incorporaría también a la gobernanza en el ámbito de las políticas públicas.

utilizadas.

27 Sobre la pérdida de soberanía estatal y nuevos modelos de tratados y acuerdos internacionales, ver (Ramos Toledano, 2016)

${ }^{28}$ El término 'gobernanza' empezó a utilizarse en los años noventa dentro del ámbito de las Relaciones Internacionales. A este respecto se suele señalar el libro Governance without Government como su vía de entrada en el mundo académico (Rosenau \& Czempiel, 1992).

Para la primera generación de estudios sobre la gobernanza es muy útil la compilación de textos realizada por Agustí Cerrillo (Cerrillo i Martínez, 2005). En relación con la segunda generación, una de las autoras de referencia es la danesa Eva Sorensen. Resulta especialmente útil a este respecto (Sorensen \& Torfing, 2006). V.t. (Torfing, 2012). 


\section{I. El experimentalismo democrático}

Como se señaló más arriba, aquí nos ocuparemos de una corriente específica de la gobernanza: el experimentalismo democrático. Se trata de un modelo que desarrollaron, entre otros, Zeitlin y Sabel ${ }^{29}$. Estos autores han escrito, además, varios trabajos sobre el Método Abierto de Coordinación (MAC) de la UE considerándolo una manifestación de esa forma de gobernanza (Zeitlin \& Sabel, 20I0) (Zeitlin, 20I4).

El referente del experimentalismo democrático es una experiencia que se inició en Chicago a finales de los ochenta y que muchos de los autores que han trabajado sobre la gobernanza (especialmente los de la primera generación de estudios) citan como modélica. La experiencia consistió en lo siguiente:

En Chicago, el sistema escolar cosechaba fracaso tras fracaso. Se habían intentado diversas políticas, unas más neoliberales, otras más sociales, pero ninguna había funcionado. La autoridad político-administrativa, los padres, los profesores y los alumnos no sabían qué hacer para evitar el fracaso escolar. Entonces se decidió poner en marcha una reforma.

La autoridad escolar fijaría unos objetivos de mejora muy generales. En cada escuela se elegiría un Consejo de Centro integrado por representantes de los profesores, los estudiantes y los padres. Cada escuela especificaría los objetivos generales y establecería el plan de estudio y la metodología para alcanzarlos. Todos los centros crearían una red horizontal para intercambiar informaciones y para poder aprender unos de otros. Cada escuela sería responsable de poner en marcha su plan y los mecanismos de control del mismo. En caso de que se viera que los objetivos no se estaban consiguiendo, se replantearían los planes y los métodos y la autoridad académica proporcionaría asistencia técnica y medios para solucionar los problemas. Al fin del curso se haría un peer review (una evaluación colectiva de los planes, métodos y resultados de cada centro) ${ }^{30}$.

Sabel acuñó la denominación 'experimentalismo democrático' para este tipo de procesos de toma de decisiones e implantación de las mismas. Lo llamaron 'experimentalismo' porque, en la experiencia que sirve de modelo, cada centro experimenta su propio proyecto y porque se sigue el método de ensayo y error. Si algo no funciona, se examinan las causas y se redefinen los planes o los medios para alcanzarlos. Lo calificaron de 'democrático' porque todos los stakeholders tienen oportunidad de participar en todas las fases del ciclo. La autoridad se limita a tareas de asistencia y a facilitar la coordinación descentralizada.

\subsection{Legitimidad}

La legitimidad del experimentalismo democrático en particular y de la gobernanza en general se basa, entre otros, en dos criterios: el de la participación de los interesados y el de la racionalidad de las discusiones. Estos criterios plantean una serie de problemas difíciles de resolver, aunque se han propuesto algunas líneas de solución a los mismos. Los principales problemas son dos:

${ }^{29}$ El primer escrito donde se formuló la teoría del experimentalismo democrático fue ("A Constitution of Democratic Experimentalism," n.d.).

${ }^{30}$ Sobre la experiencia de las escuelas de Chicago (Sabel, 2002). 
I. ¿Cómo se puede contrarrestar el diferente poder de negociación de los stakeholders (por ejemplo una multinacional y un grupo ecologista local) en el caso de la instalación de una fábrica contaminante? (Santos \& Rodríguez Garavito, 2007).

2. ¿Cómo se puede conseguir que los stakeholders (especialmente las empresas) adquieran realmente la predisposición de intercambiar argumentos para encontrar la mejor solución al problema en lugar de limitarse a defender sus intereses utilizando su poder de negociación? (Estévez Araujo, 2008).

La cuestión de la fundamentación de los criterios de legitimidad de la gobernanza se ha abordado más profundamente en otros trabajos y es un tema en el que seguimos investigando. Aquí únicamente intentamos comprobar si las pretensiones de legitimidad se cumplen en la práctica, en base a los propios criterios del experimentalismo democrático. Más adelante especificaremos en un sentido más preciso qué entendemos aquí por 'participación' y por 'racionalidad'.

\subsection{El Método Abierto de Coordinación}

El Método Abierto de Coordinación de la UE fue considerado como una forma de experimentalismo democrático, tal y como ya se ha dicho. El MAC se institucionalizó en el año 2000 en el marco de la Estrategia de Lisboa, aunque había habido algunas experiencias previas en el marco de las políticas de empleo en los noventa.

En principio el MAC estaba pensado especialmente para las políticas sociales. La UE no tiene prácticamente ninguna competencia en esa materia y las pocas que tiene requieren acuerdos unánimes para poder tomar decisiones, por lo que cualquier estado puede vetarlas. Lo que se pretendía con el MAC en el ámbito de las políticas sociales era que los estados de la UE intercambiasen informaciones y experiencias de modo que la visión de los problemas en materia de política social pudiera irse aproximando. Se trata, por ello, de un método de 'coordinación', no de armonización. ${ }^{31}$

EI MAC se planteaba como algo completamente distinto e independiente del Método Comunitario (MC). El MC es el conjunto de procedimientos por medio de los cuales los órganos de la UE (Consejo, Comisión, Parlamento, Tribunal de Justicia) aprueban normas y adoptan resoluciones. La mayor parte de ellas (aunque no todas) son vinculantes para los estados miembros y para las propias instituciones de la Unión. En cambio, el objetivo del MAC era la aproximación de perspectivas en relación con las políticas sociales ente los estados miembros y únicamente utilizaba mecanismos de soft law, nunca de hard law.

EI MAC constaba de las siguientes fases:

I) Los estados miembros (a través del Consejo) definían los objetivos generales a alcanzar, los indicadores y las líneas directrices.

2) Los Planes de Acción Nacional (PAN) eran elaborados por cada uno de los estados que querían participar en el MAC. En ellos se señalaba el nivel que había alcanzado en la

\footnotetext{
${ }^{31}$ Armonizar las políticas sociales hubiera significado aprobar directivas o reglamentos para que los estados regulasen las políticas sociales de acuerdo con lo establecido en los mismos.
} 
consecución de los objetivos de acuerdo con los indicadores. También se incluían los cambios propuestos para mejorar los resultados.

3) Se realizaban procesos de peer review de los planes y elaboración de informes y recomendaciones por parte de la Comisión Europea (CE).

4) Reelaboración periódica de los PAN's (cada año o cada dos años según el proceso de que se trate) $y$, en intervalos mayores, de los objetivos e indicadores a nivel europeo en función de la experiencia obtenida por su puesta en práctica.

\section{El Semestre europeo y los mecanismos de coordinación de las políticas contra la pobreza}

En la Estrategia Europa 2020, aprobada en 2010, el MAC desapareció del mapa. En dicha estrategia se establecen una serie de objetivos sociales (como sacar de la pobreza a 20 millones de personas), pero durante el periodo 2010-20I I pareció darse por finiquitado el MAC. Sin embargo, a partir de un documento presentado por el Comité de Protección Social (CPS) en mayo-junio de 20II (CPS, 20II) se decidió reinstaurar el MAC, pero éste quedó incluido total, o al menos parcialmente en el llamado Semestre Europeo.

\section{I. ¿Qué es el Semestre Europeo (SE)?}

El semestre europeo es una arquitectura institucional que se ha instaurado por la estrategia Europa 2020. El término 'arquitectura' se ha popularizado en base al trabajo de Susana Borrás y Claudio Radaelli (Borrás \& Radaelli, 20I I). Una 'arquitectura' tiene un rango intermedio entre los Tratados y las decisiones ordinarias adoptadas por los órganos de la Unión Europea. En ella se incluyen principios de actuación, objetivos estratégicos, métodos específicos de toma de decisiones... Todo ello en principio sin alterar el contenido de los Tratados. Aparte de la estrategia Europa 2020, la estrategia de Lisboa (que comprendió el período 200I-2010) es otro ejemplo de arquitectura europea.

El Semestre Europeo es un ciclo que se inicia en octubre de un año (por ejemplo 2014) y que finaliza en diciembre del año siguiente (p.ej. 2015). Sus hitos más importantes son los siguientes:

I) El informe anual prospectivo de crecimiento. El proyecto es elaborado por la Comisión Europea en octubre-noviembre y presenta un panorama general de la situación en la Unión y de las líneas de actuación de las diferentes políticas públicas. La propuesta de la Comisión Europea es examinada y enmendada por los órganos consultivos y por el Consejo de Ministros $^{32}$, y finalmente el Consejo Europeo aprueba el documento en marzo.

2) Las recomendaciones específicas para cada país. Éstas se elaboran en base al informe prospectivo. La propuesta la realiza la Comisión Europea. Esta propuesta es objeto de diversos dictámenes de los órganos consultivos y decisiones de las diferentes

\footnotetext{
${ }^{32} \mathrm{El}$ Consejo de Ministros tiene diversas formaciones en función del tema de que se trate. En el ámbito de lucha contra la pobreza e inclusión social, se ocupa el EPSCO (Empleo, Política Social, Sanidad y Consumidores).
} 
formaciones del Consejo de Ministros. Finalmente son aprobadas por el Consejo Europeo en junio-julio.

3) Programas Nacionales de Reforma: los elabora cada uno de los estados miembros en base a lo establecido en el informe prospectivo anual y en las recomendaciones específicas que ese estado haya recibido. Estos documentos y, eventualmente, el Informe Nacional Social (ver más adelante) son presentados a las instancias europeas durante el mes de abril.

4) Evaluación de los informes y de los logros alcanzados en el ámbito de las diferentes políticas por parte de los organismos europeos y por medio también de procedimientos de revisión por pares.

5) En el caso de los países de la Eurozona: los Estados presentan sus proyectos de presupuestos a la CE (septiembre); la Comisión formula opiniones sobre los proyectos de presupuesto (noviembre); el Consejo Europeo discute acerca de las opiniones de la $\mathrm{CE}$ (diciembre-enero). Todo ello constituye una especie de examen previo. Es necesario que los Estados de la Eurozona lo realicen para poder aprobar sus presupuestos para el siguiente año (diciembre-enero) ${ }^{33}$.

Es obvio que existen grandes similitudes entre el MAC y el SE. La diferencia fundamental consiste en que la participación que en el MAC era voluntaria, mientras que los estados están obligados a tomar parte en el Semestre Europeo.

\subsection{Contextualización de la coordinación de las políticas sociales en el marco del_SE}

Hay que situar el problema de la coordinación de las políticas sociales a nivel europeo en un contexto más amplio. Este contexto puede ser representado como una serie de círculos concéntricos.

\footnotetext{
${ }^{33}$ Todo lo relativo a la Estrategia Europa 2020 y al Semestre Europeo puede experimentar cambios importantes cuando se apruebe el Mid Term Review. La evaluación tenía que haber finalizado en 2015, pero en el momento de escribir estas líneas (abril de 2016) todavía está en proceso de elaboración. En cualquier caso, una de las cosas que podría suceder es que se eliminase la cuantificación de los objetivos de la lucha contra la pobreza, que suscitó muchas reticencias en varios países (Jessoula, 2015).

En el Marco del Semestre Europeo se lleva a cabo otro proceso simultáneo al aquí descrito. Se trata del Procedimiento de Desequilibrios Macroeconómicos (PDM). Si se establece que existe un desequilibrio no excesivo, el seguimiento se produce en el brazo preventivo del PDM. Las recomendaciones del PDM se integran en el paquete de propuestas de recomendaciones específicas para cada país.

Si el desequilibrio es considerado excesivo (pudiendo perjudicar el funcionamiento de la Unión Económica y Monetaria) la Comisión activa el brazo correctivo del PDM: el Procedimiento de Desequilibrios Excesivos. El Estado miembro en cuestión deberá presentar un plan de 'acción correctiva'. La Comisión llevará a cabo un seguimiento periódico. La aplicación del Procedimiento de Desequilibrios Excesivos para los Estados miembros de la zona del euro conlleva sanciones (de hasta el $0,1 \%$ del PIB) en caso de que no se tomen las medidas acordadas en repetidas ocasiones o no se presente un plan de acciones correctivas adecuado. Sin embargo, el Procedimiento de Desequilibrio Excesivo todavía no se ha implementado en ninguna ocasión.

Los países con un programa de rescate (como Grecia o Irlanda) no se incluyen en el Procedimiento de Desequilibrios Macroeconómico, dado que ya cuentan con una supervisión económica estricta vinculada a la ayuda financiera recibida en el marco del Mecanismo Europeo de Estabilidad.

La Comisión Europea señala en los Documentos relativos al Semestre Europeo 2016 que, en el caso de España, se han detectado desequilibrios 'excesivos' que nos sitúan en el nivel 5 de los 6 establecidos como categorías de los desequilibrios. Nos encontramos, así, en el límite con las situaciones que darían pie al inicio del procedimiento de desequilibrio excesivo (PDE), al que, como se ha señalado, todavía no se ha recurrido nunca.
} 
El círculo exterior lo constituye el proceso de globalización neoliberal, la crisis financiera de 2008, y las repercusiones de esa crisis en la Unión Europea y especialmente en la Eurozona. El segundo círculo estaría constituido por las orientaciones neoliberales de las políticas anti-crisis adoptadas en Europa. El tercer círculo configura el entramado institucional de la UE y los nuevos mecanismos formales e informales que se han puesto en marcha para la gestión de la crisis (Mecanismo Europeo de Estabilidad, cartas -no tan secretas- del Banco Central Europeo, Eurogrupo, reuniones bilaterales de los estados con la Comisión y con Alemania, etc.). El cuarto círculo lo constituye el Semestre Europeo dentro del que se realiza la coordinación de las políticas sociales en el marco de la estrategia Europa 2020. Y por último, el quinto, correspondería a la coordinación de las políticas de lucha contra la pobreza.

Los primeros tres círculos actúan como corazas. Constriñen de forma implacable la posibilidad de desarrollar políticas sociales a nivel de la Unión Europea y, sobre todo, a nivel de los estados. El cuarto círculo puede actuar como una constricción o, por el contrario, como una ampliación. Eso depende de varios factores, por ejemplo de que se considere que la creación de empleo es el único y exclusivo camino para garantizar la salida de la pobreza. Desde nuestro punto de vista es necesario aceptar la necesidad de políticas asistenciales además de las de empleo para disminuir la pobreza o el riesgo de caer en ella. ${ }^{34}$

\section{Cuadro I: contextualización e las políticas de lucha contra la pobreza}

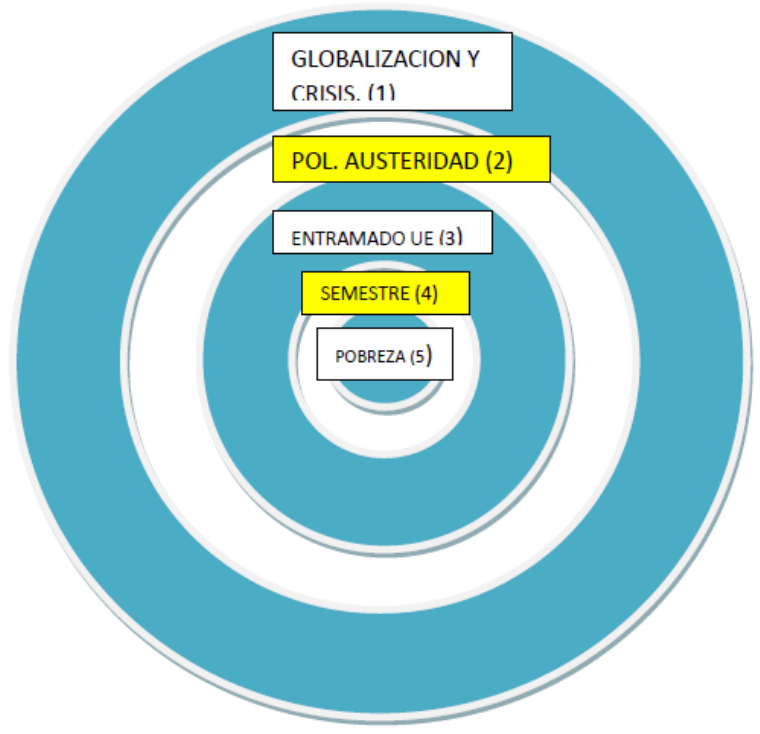

\subsection{El Comité de Protección Social (CPS)}

El Comité de Protección Social es un organismo clave en la coordinación de las políticas sociales de lucha contra la pobreza en la Unión Europea. A través de él se canalizan los diversos documentos nacionales. Participa en la toma de decisiones de todas las fases

\footnotetext{
${ }^{34}$ Sobre las inconsistencias del diseño de las políticas de lucha contra la pobreza y la exclusión social, ver Copeland et al., 2014.
} 
del semestre. Es un órgano consultivo de la Comisión, del Consejo de Ministros encargado de asuntos sociales (EPSCO) y del propio Consejo de Europa.

El Subgrupo de Indicadores es un órgano especialmente importante del Comité de Protección Social. El Subgrupo de Indicadores selecciona los diferentes datos que van a tenerse en cuenta. Los indicadores construidos a partir de ellos servirán por ejemplo para medir la pobreza. Las discusiones de este subgrupo son formalmente técnicas, pero en la práctica hay un importante componente de negociación política, aunque ésta no sea explícita. En realidad, el Comité no está formado por expertos independientes. Está integrado por expertos nombrados por los países miembros. Cada uno de ellos intenta que se aprueben indicadores que favorezcan los índices de su propio país.

El Subgrupo de Indicadores opera dentro de la opacidad propia del funcionamiento general del Comité de Protección Social. Pero además está totalmente 'blindado'. No hay ningún tipo de participación de las organizaciones sociales en sus sesiones. Eso es así a pesar de la importancia que tiene la configuración de los indicadores, pues éstos son clave para definir las políticas sociales y evaluar sus resultados. Estamos ante uno de esos frecuentes casos en que decisiones con un componente político se presentan como puramente técnicas para dejarlas exclusivamente en manos de 'expertos'. De esa forma se 'justifica' la no participación de los ciudadanos en la toma de dichas decisiones.

El funcionamiento de las sesiones del CPS es poco transparente ${ }^{35}$. No hay convocatorias formales, no se levantan actas de las sesiones... Las decisiones se han tomado tradicionalmente por consenso. Sin embargo, recientemente se han introducido votaciones en casos puntuales. Es especialmente importante la introducción de votaciones en los dictámenes acerca de las propuestas de recomendaciones específicas que hace la Comisión. Si el Comité de Protección Social vota en contra por mayoría cualificada reversa, la Comisión debe modificar su propuesta.

\subsection{Legitimidad: democracia y racionalidad}

Para evaluar la legitimidad de los procesos de toma de decisiones en base al criterio de la democracia, utilizaremos un esquema que contiene una tipología de formas de participación. A cada forma le hemos asignado un grado de intensidad en una escala. Hemos elaborado el cuadro a partir de un informe elaborado en 2012 por la European Anti Poverty Network (EAPN). En él se analizaban, entre otras cosas, los mecanismos de participación en los Programas Nacionales de Reforma y los Informes Nacional Sociales (EAPN, 20I2).

La racionalidad de los procesos de toma de decisiones es una cuestión de grado. Se encuentra en algún punto de una línea cuyos dos extremos podríamos denominar 'deliberación' y 'negociación'. La negociación es un 'juego del lenguaje' en el que las partes implicadas adoptan una actitud de defensa a ultranza de sus propios intereses. Pueden realizarse transacciones entre los mismos, pero de acuerdo con una lógica de do ut des. El contenido de las transacciones y la decisión final vienen determinados por

\footnotetext{
35 Hay muy pocos trabajos sobre el Comité de Protección Social en castellano o en inglés. Las informaciones aquí reflejadas se han obtenido de la tesis de Anna Horváth (Horváth, 2009) y de las reuniones con los funcionarios del Ministerio de Sanidad Seguridad Social e Igualdad en febrero de 2016.
} 
la correlación de fuerzas existente entre las partes. Entre estas 'fuerzas' hay que contar diversos tipos de 'capital': económico, político, pero también capital cultural jurídico y simbólico. En el 'juego' de la deliberación la actitud de los participantes es diferente. Su objetivo es encontrar la mejor solución al problema de que se trate. Para ello se atienen a las reglas de la argumentación, especialmente a la veracidad y a la probidad.

Los juristas franceses llaman en ocasiones Droit Negocié a lo que aquí denominamos 'gobernanza'. En sus análisis se señala que los procesos de toma de decisiones son más racionales que la negociación. No consisten en una transacción entre intereses determinada por la correlación de fuerzas. Pero su racionalidad no alcanza el grado de la deliberación. ${ }^{36}$

\section{Cuadro 2: formas y niveles de participación}

\begin{tabular}{|c|c|}
\hline INTENSIDAD & CONTENIDO \\
\hline$\underline{1}$ & $\begin{array}{l}\text { Envío de cartas, informes, valoraciones, etc. a las autoridades por } \\
\text { iniciativa propia. }\end{array}$ \\
\hline$\underline{2}$ & $\begin{array}{l}\text { Consulta: requerimiento por escrito de opiniones, valoraciones y } \\
\text { propuestas por parte de los organismos oficiales }\end{array}$ \\
\hline$\underline{3}$ & $\begin{array}{l}\text { Discusión: participación en reuniones con representantes de otros } \\
\text { grupos y de la administración y las que se intercambian opiniones, } \\
\text { argumentos, informaciones, etc. } \\
\text { 3.1 Discusiones puntuales e informales } \\
\text { 3.2 Discusiones que tienen lugar periódicamente }\end{array}$ \\
\hline$\underline{4}$ & $\begin{array}{l}\text { Establecimiento de una estructura organizativa para la discusión y } \\
\text { para el mantenimiento de un contacto continuado. }\end{array}$ \\
\hline$\underline{5}$ & $\begin{array}{l}\text { Toma de decisiones: derecho de influir en la decisión final que se } \\
\text { adopte sea esta por consenso o por votación. } \\
\text { 5.1. Participación en la toma de decisiones de órganos consultivos no } \\
\text { vinculantes. } \\
\text { 5.2. Participación en la toma de decisiones de órganos consultivos } \\
\text { vinculantes. } \\
\text { 5. 3. Participación en la formulación de las resoluciones de órganos } \\
\text { ejecutivos o con poder normativo. }\end{array}$ \\
\hline
\end{tabular}

La participación de las organizaciones sociales en el CPS no supera la intensidad del grado 3.I. Únicamente se realizan discusiones puntuales con representantes de las organizaciones que trabajan en el campo de la integración social. Esa participación se canaliza fundamentalmente a través de una organización paraguas: la Plataforma Social Europea. Dentro de ésta, así como entre las organizaciones europeas en general, tiene especial importancia para nuestro trabajo la Plataforma Europea de Lucha contra la Pobreza (EAPN en sus siglas en inglés). ${ }^{37}$

\footnotetext{
${ }^{36}$ V. p. ej. Gérard, Ost, \& Kerchove, 1996.

${ }^{37}$ En una intervención ante el CPS del Presidente de la Plataforma Social Europea, Heather Roy, llevada a cabo el 17 de diciembre de 2014 éste se quejaba, entre otras cosas, de no haber podido participar en conjuntamente con los interlocutores sociales.

La Red europea de lucha contra la pobreza (EAPN) ha incentivado otras formas de participación en el Semestre Europeo. Lo ha hecho creando la Semester Alliance. Esta organización ha editado una serie de
} 
¿El grado de racionalidad de las reuniones del Comité de Protección Social es muy diferente en el primer semestre y en el segundo. Los primeros seis meses del año vienen marcados por la elaboración de informes, dictámenes, opiniones y otros documentos relacionados con la mecánica del Semestre Europeo. En ese caso predomina la negociación ${ }^{38}$ entre los estados y, especialmente, entre éstos y la Comisión Europea. En la segunda parte del año se realizan una serie de procesos de review. En este caso el juego del lenguaje que se practica es más deliberativo. Los representantes de los estados están ciertamente interesados en encontrar la mejor solución posible a los problemas que se plantean. Su actitud no es la de defender los intereses de los gobiernos que representan. Es una postura que implica una disposición a aprender (learning), tanto de las buenas prácticas de otros estados como de las observaciones críticas que se realizan sobre las prácticas propias ${ }^{39}$.

\section{Los Programas e Informes Nacionales del Reino de España}

En el ámbito del Semestre Europeo, hay determinados documentos elaborados por los estados que resultan de importancia a la hora de entender qué medidas se proponen y aplican (y quién está legitimado para intervenir en el proceso) con el objetivo de reducir el riesgo de pobreza y exclusión social de determinados sectores de población. Destacan, tanto porque señalan la línea programática teórica a seguir, como por su importancia a la hora de determinar las políticas que lleva a cabo un país, el Plan Nacional de Acción para la Inclusión Social (PNAIN 2013-2016) y los Programas Nacionales de Reformas. Y, en menor medida (entre otras cosas por su carácter voluntario), los Informes Nacional Sociales.

EI PNAIN ${ }^{40}$ es un documento de carácter genérico en el que se plantean los objetivos y acciones principales a llevar a cabo por un país en el ámbito de la inclusión social. En

materiales didácticos sobre el funcionamiento del semestre europeo y sobre los mecanismos que las ONGs tanto locales como europeas, pueden utilizar para incidir en él. (V.http://semesteralliance.net/)

${ }^{38}$ En relación con el tema de la negociación, Habermas sostiene que la ese juego del lenguaje da como resultado arreglos o compromisos ("Schlichtung" o "Kompromißbildung” en el original alemán), mientras que lo que aquí denominamos "deliberación” da lugar a consensos ("Konsens”) (Habermas, 1998, pp. 207208) (Habermas, 1994 p. 176).

Nosotros consideramos que, en principio, la negociación no es un juego de suma cero. No obstante, pueden darse casos en que una negociación concreta resulte ser un juego de ese tipo. En determinados casos se puede afirmar p. ej. que ha habido una negociación cuyo resultado ha beneficiado únicamente a una de las partes en perjuicio de la otra. No compartimos, pues, la opinión que todos los casos en que las negociaciones acaban convirtiéndose en un juego de suma cero deban considerarse como un 'fracaso'. En contra, Weiss, 2014.

En cuanto a la deliberación existen diversas formulaciones de sus reglas (Atienza, 2013).

${ }^{39}$ En el marco del CPS se llevan a cabo diversos tipos de peer reviews: las revisiones temáticas, las revisiones ex ante, los peer reviews ad hoc y las revisiones multilaterales. Estas últimas se inscriben dentro del SE. No obstante, de acuerdo con las informaciones recogidas, la dinámica de las sesiones en este caso es más deliberativa que negociadora (entrevista MSSSI febrero 2016). Ver también Zeitlin, 2014. Por insuficiencia de espacio no podemos exponer aquí en qué consisten todos estos tipos de reviews. Sin embargo, más adelante, al hablar de la elaboración de los documentos españoles destinados al SE, describiremos un caso de peer review ad hoc.

${ }^{40}$ Respecto a la creación del Plan Nacional, las Recomendaciones Específicas de 2013, en su considerando 14, aludían a la falta de información sobre el proceso de creación del PNAIN 2013-2016, pero no recomendaban de forma específica que éste fuera creado. En las CSR de 2012, la recomendación 7 hablaba de medidas específicas para combatir la pobreza, pero sin más concreción. Tampoco en los PNR de 2012 ni 2013 se hace mención alguna al PNAIN, como sí sucede en el de 2014. Sin embargo, el Informe de Seguimiento del PNAIN afirma que éste está basado en el enfoque de la Inclusión Activa, adoptado por 
este sentido, responde a los objetivos marcados en la Estrategia Europa 2020, entre los que se encuentra la reducción en 20 millones las personas en riesgo de pobreza y exclusión social en toda la UE. Para España, el objetivo oscila entre I,4 y I,5 millones.

El Plan establece tres objetivos principales, denominados objetivos estratégicos, que a su vez prevén diferentes objetivos y, éstos, actuaciones, que son propuestas encaminadas a alcanzar los objetivos que, juntos, conforman cada objetivo estratégico. El primero de ellos -en consonancia con la tendencia de las instituciones de la UE a apostar por el empleo como mecanismo principal para evitar la pobreza y la exclusión social- hace clara referencia al empleo como mecanismo de inclusión de las personas más vulnerables. Es más, el propio plan considera absolutamente complementarias tales medidas a la flexibilización de las condiciones laborales -que en muchos casos no han supuesto sino una reducción de los derechos de los trabajadores- y a la llamada «flexiguridad». El segundo objetivo estratégico hace referencia a los sistemas de prestaciones «que no desincentiven el trabajo». Finalmente, el Plan establece los objetivos en relación a la prestación de servicios públicos básicos, como los servicios sociales, la educación o la sanidad.

EI PNAIN es, por tanto, un documento relevante porque plasma la estrategia a seguir por un país durante los años siguientes en lo que a riesgo de pobreza y exclusión social se refiere. Su redacción permite, asimismo, la creación de un marco de trabajo con un ámbito tan relevante como el de las ONG y las entidades del Tercer Sector, que conocen bien a los sectores de población a los que se dirige el PNAIN. Su participación resulta, pues, de vital importancia para que las propuestas del Plan resulten lo más efectivas a la hora de alcanzar el objetivo de reducir en I,5 millones las personas en riesgo de pobreza y exclusión social.

El Programa Nacional de Reformas (PNR, 2015) es el documento más importante de los que se elaboran a nivel estatal. Se trata de un proyecto de actuación que los estados deben presentar obligatoriamente en el marco del Semestre Europeo. Se elabora en base al Informe Anual Prospectivo de Crecimiento y debe dar respuesta a las Recomendaciones Específicas que se hayan hecho al país en cuestión. Por ello abarca todas las políticas públicas, entre ellas, las sociales. Es, pues, el documento que más claramente manifiesta las intenciones de un país respecto a elementos como el mercado de trabajo, el déficit fiscal o las políticas sociales, aunque constreñidas aquéllas a lo determinado por el Consejo Europeo mediante sus Recomendaciones. Aunque el MSSSI participa en su creación con aportaciones y propuestas, la coordinación corresponde a la Oficina Económica de la Presidencia del Gobierno.

Como se ha afirmado con anterioridad, el Semestre Europeo coordina las políticas sociales de los estados, y en este marco la creación de empleo se plantea como la principal forma de evitar la exclusión social y la pobreza. Pero ello conlleva el riesgo de desatender otros dos de los tres pilares fundamentales en la lucha contra la pobreza que se plasmaron en el PNAIN 2013-2016 (empleo, prestaciones sociales y servicios públicos). Así, las medidas adoptadas por los estados en el marco de la estrategia Europa 2020, una de cuyas aspiraciones es la reducción de la pobreza en 20 millones de personas en la Unión Europea, pueden resultar contraproducentes para tal objetivo precisamente

una Recomendación de la Comisión de 2008. 
por focalizar los esfuerzos en el ámbito laboral ${ }^{41}$ a la vez que se reducen los ámbitos de intervención pública en lo que a prestaciones y servicios públicos se refiere.

En este contexto, no es de extrañar que las referencias en el PNR a medidas concretas de lucha contra la pobreza y la exclusión social sean mínimas. EI PNR debe dar respuesta a las Recomendaciones Específicas del Consejo, $y$ en éste ninguna de las recomendaciones va directamente dirigida a las medidas de carácter social. A lo sumo, la referencia es a la creación de empleo y a la aplicación del PNAIN 2013-2016 (recomendación $\mathrm{n}^{\circ} 5$ ).

En consonancia, el PNR justifica el aumento de pobreza por las elevadas tasas de desempleo, obviando que medidas de intervención pública como las prestaciones o los servicios públicos pueden tener una incidencia directa en el riesgo de exclusión social de la población. En este sentido, el PNR justifica su cumplimiento de las Recomendaciones Específicas haciendo referencia a las políticas de empleo y a la reforma educativa llevadas a cabo durante 2014 y a la elaboración de distintos Planes como el PNAIN.

Otro de los documentos relevantes son los llamados Informes Nacional Sociales (INS, 2012 y 20l4). Se trata de documentos voluntarios (los estados pueden decidir si presentarlos o no) que elaboran conjuntamente el Ministerio de Sanidad, Servicios Sociales e lgualdad y el Ministerio de Empleo y Seguridad Social. Tienen carácter bienal, y son uno de los elementos del Método Abierto de Coordinación en sentido estricto que se ha mantenido en el marco del Semestre Europeo.

La importancia de estos documentos -complementarios a los PNR- en el marco de la investigación aquí desarrollada, deriva de su exposición detallada de las políticas sociales llevadas a cabo por un país (en los PNR, la mención es mínima). Los INS, en este sentido, especifican los programas de lucha contra la pobreza y la exclusión social y los avances conseguidos respecto a la Estrategia Europa 2020.

\section{Participación ${ }^{42}$}

En el ámbito de la pobreza y la exclusión social, las ONG y entidades del Tercer Sector son actores fundamentales. Los propios documentos españoles (PNAIN, INS, Informes de Seguimiento e incluso el PNR) así lo reconocen, y hacen hincapié en la importancia de la colaboración público-privada. ${ }^{43}$ Por ello, resulta fundamental determinar en qué

\footnotetext{
${ }^{41}$ Otro debate (aunque no enteramente desligado) se plantea al analizar cómo la obtención de un empleo no necesariamente implica el fin de la situación de pobreza o exclusión social. La degradación de las condiciones laborales ha provocado que existan hogares en los que, pese a que al menos una persona trabaja, no se supera el umbral de pobreza. Los trabajadores pobres han aumentado en toda la Unión Europea en las últimas dos décadas, si bien en España el aumento ha triplicado la media europea, según datos de Eurostat. Ver Eurostat 'in-work at-risk-of-poverty', en European Union Statistics on Income and Living Conditions (EU-SILC).

${ }^{42} \mathrm{La}$ información relativa a la participación es fruto, principalmente, del análisis de los propios documentos españoles y sobre España y de las reuniones mantenidas con el Ministerio de Sanidad, Servicios Sociales e Igualdad en febrero de 2016. Las reuniones, encuestas y entrevistas mantenidas con las ONG y entidades del Tercer Sector, que nos permitirán completar adecuadamente este apartado, se encuentran actualmente en curso.

${ }^{43}$ En prácticamente cada objetivo del PNAIN, se prevé como mínimo una actuación que haga referencia al Tercer Sector, en términos de «desarrollar, en colaboración», «contando con el apoyo de» $\mathrm{O}$ «cooperar con»
} 
medida las ONG y entidades intervienen en la elaboración y aplicación de estos documentos, lo que sin duda incide en la legitimidad democrática de las políticas sociales llevadas a cabo por un país.

La colaboración público-privada descrita en los documentos españoles puede tener varias vertientes. En este sentido, los planes e informes reconocen como sujeto colaborador al sector empresarial, e incluyen la Estrategia Española de Responsabilidad Social de las Empresas 2014-2020 en el marco de los avances en la lucha contra la pobreza y la exclusión social. ${ }^{44}$

Sin embargo, son las ONG y entidades del Tercer Sector las consideradas como actor principal en los objetivos de reducción de la pobreza y exclusión social. Por ello, la propia elaboración de un documento como el PNAIN contó con una importante participación por parte de las entidades. En una primera reunión, a principios de 2013 , entre el representante del MSSSI y el Grupo de Trabajo sobre Inclusión Social, Empleo y Rural ${ }^{45}$, se expuso por parte del representante ministerial el planteamiento, esquema, objetivos y plazos del PNAIN. Posteriormente las ONG y entidades pudieron participar en un turno de palabras y obtuvieron unas fichas a rellenar de forma que pudieran enviarlas al MSSSI aportando sus propuestas. Éstas fueron, según el Ministerio, tenidas en cuenta a la hora de realizar el PNAIN, incorporándolas al borrador, que fue presentado en otra reunión (a finales de 2013). En esta segunda reunión las ONG y entidades valoraron a nivel técnico y de contenido el borrador que les había sido presentado.

La intensidad de la participación en la elaboración y seguimiento de los diferentes informes y programas en España varía mucho de unos a otros. Va desde la ausencia de canales de participación (caso de los Informes de Seguimiento del PNAIN), pasando por la consulta (nivel 2 de intensidad) hasta la participación en la toma de decisiones de órganos consultivos no vinculantes (nivel 5.I de intensidad), como en el caso del PNAIN, que cuenta con el grado máximo de participación. En cambio, los Informes Nacional Sociales cuentan con una participación mínima, dado que se trata de una valoración por parte del propio Estado de los avances respecto a los objetivos marcados en los PNR.

Las estructuras de participación permanente (como las ONG y entidades, o el Grupo de Trabajo sobre Inclusión Social, Empleo y Rural) están estrechamente vinculadas con el MSSSI, pero las decisiones que se adoptan finalmente por parte del Ministerio no quedan condicionadas por sus informes. Por otro lado, las propias decisiones del MSSSI pueden ser anuladas o modificadas por otros ministerios y por la Presidencia del Gobierno. Eso ocurrió por ejemplo con el último INS, que no pudo presentarse a los organismos europeos.

El hecho de que existan estructuras permanentes de diálogo, que permiten un nivel de participación de intensidad 5.I, debió ser la razón de que La EAPN pusiera como modelo de gobernanza a España el año pasado. Lo hizo precisamente en lo que se refiere a la participación de las entidades sociales. El Presidente del Consejo Europeo de Protección

\footnotetext{
el Tercer Sector.

${ }^{44}$ Informe de Seguimiento del PNAIN 2013-2016, p. 16.

${ }^{45}$ El Grupo de Trabajo sobre Inclusión Social, Empleo y Rural es el principal canal de participación de las ONG y el Tercer Sector en la creación del PNAIN. Fue creado en el seno del Consejo Estatal de ONG de Acción Social, órgano consultivo adscrito a la Administración General del Estado a través del MSSSI.
} 
Social propuso que el Estado español organizase un peer review ad hoc sobre ese tema. Finalmente, no se pudo por la inmensa acumulación de trabajo en el seno de la Subdirección General de Programas Sociales del MSSSI.

\section{Eficacia}

La eficacia de los mecanismos de gobernanza se evalúa en diversos frentes. Uno de ellos se refiere a la efectividad de los mecanismos de soft law. Otro tiene que ver con la efectiva consecución de los objetivos. En este caso se trata de sacar a 20 millones de personas de la pobreza en Europa, de las cuales I millón y medio corresponden a España.

El efecto de shame es uno de los elementos que se señalaban como bases de la eficacia de los mecanismos de soft law en el marco del MAC. La 'vergüenza' se refiere al hecho de ser señalado con el dedo por parte de la Comisión. En este sentido tienen una especial importancia las Recomendaciones Específicas por país. De acuerdo con las impresiones obtenidas de las entrevistas realizadas en el Ministerio, la reacción psicológica frente a las mismas nos pareció bastante intensa.

Así, una de las Recomendaciones Específicas relativas a España aprobadas en 2015 se refería a los sistemas de rentas mínimas. España tiene 19 sistemas diferentes de este tipo de rentas. La Recomendación se refería a la necesidad de racionalizar y coordinar mejor todos esos sistemas:

RECOMENDACIÓN DEL CONSEJO de 14 de julio de 2015 relativa al Programa Nacional de Reformas de 2015 de España y por la que se emite un dictamen del Consejo sobre el Programa de Estabilidad de 2015 de España

Considerando 13: España ha realizado avances limitados en la mejora de la eficacia de su sistema de protección social. Ha establecido un nuevo programa de activación para los desempleados de larga duración, que combina el apoyo a la renta con asistencia en la búsqueda de empleo. No obstante, la coordinación limitada entre los servicios de empleo y los servicios sociales y la incompatibilidad de los distintos regímenes de renta mínima han reducido la eficacia de los programas de ayuda social. Afrontando unos altos niveles de pobreza, especialmente entre los hogares de baja renta con hijos, España ha realizado avances limitados en la mejora de la orientación específica de los sistemas de ayuda familiar y los servicios asistenciales.

Recomendación Específica: Racionalizar los regímenes de renta mínima y de ayuda familiar.

Esa Recomendación fue recibida con especial preocupación en la Subdirección, acompañada de una cierta irritación. Ésta venía motivada por el hecho de que la Comisión no conocía la información contenida en el último INS. Y la desconocía porque dicho Informe no llegó a enviarse: como se ha señalado antes, fue 'vetado' por las instancias gubernamentales superiores o por la propia Presidencia del Gobierno.

De haberlo recibido, la Comisión hubiera sabido que el mayor porcentaje del presupuesto en materia de rentas mínimas lo gestiona el Estado. Las CCAA se ocupan 
del 10\%-15\% del total. No obstante, desde la Subdirección se reconoce que los programas de rentas mínimas no están bien coordinados y algunas prestaciones se solapan. También hay personas que quedan fuera de todos los sistemas de prestaciones.

En relación al objetivo cuantificado de reducción de la pobreza, la ineficacia tanto a nivel estatal como europeo resulta patente. En el conjunto de la Unión Europea la pobreza ha aumentado en 8 millones de personas, por lo que estamos a $\mathbf{- 2 8 . 0 0 0 . 0 0 0 ~ d e ~ d i s t a n c i a ~}$ de conseguir el objetivo previsto en la Estrategia 2020. En el caso español, no tenemos datos sobre el número absoluto de personas pobres. Pero su porcentaje en relación con la población total se ha mantenido igual según determinadas estadísticas y aumentado considerablemente según otras. En cualquier caso no parece haberse realizado progreso alguno en lo relativo a sustraer personas de la condición de pobreza o del riesgo de caer en la misma.

\section{Conclusiones}

El estudio del discurso y la práctica de la gobernanza es especialmente importante para los juristas y los filósofos del derecho. La gobernanza disputa la legitimidad a la democracia representativa. Se presenta como una forma de democracia participativa que complementa, o incluso debería sustituir a los mecanismos representativos. Además, buena parte de la generación del derecho en la actualidad se realiza por medio de mecanismos de gobernanza. Esto es especialmente cierto en el caso de la Unión Europea. No sólo en el ámbito del MAC o del Semestre Europeo, sino también en el marco del llamado Método Comunitario. En base a los estudios realizados y a las conclusiones provisionales obtenidas en la presente investigación, podemos afirmar que la gobernanza provoca una privatización de la generación del derecho. Tanto lo que se llama "creación", como lo que se denomina "aplicación" del derecho, se transfiere a las entidades privadas con mayor poder económico.

El grado de legitimidad de los procedimientos de toma de decisiones en el marco de la coordinación de políticas sociales de la UE es muy bajo. Hay que tener en cuenta las constricciones a las que están sometidas. Como se pone de manifiesto en el cuadro I (contextualización e las políticas de lucha contra la pobreza), el margen de maniobra que tienen los organismos de la Unión Europea encargados de la coordinación de las políticas sociales y los propios estados es enormemente restringido. Por otro lado, el grado de participación de las ONGs en un órgano clave como es el Comité de Protección Social es extraordinariamente bajo de acuerdo con las conclusiones provisionales de la investigación. En cuanto a la racionalidad de las discusiones en el marco del CPS, la hemos analizado de forma separada a la participación por razones de claridad y refiriéndonos exclusivamente a las reuniones de los representantes de los estados y la Comisión. Sin embargo el grado de apertura de las discusiones debería ser considerado también un criterio de racionalidad. El resultado de la combinación de ambos criterios estudiados por separado daría como resultado un grado global de legitimidad mucho menor.

Respecto de los documentos relativos a España, uno de los objetivos principales de este trabajo ha sido analizar el nivel de participación de ONG y entidades del Tercer Sector en la elaboración y aplicación de los mismos. Es decir, con qué grado de legitimidad cuentan los informes y programas respecto a los colectivos a los que afectan en el marco 
del Semestre Europeo ${ }^{46}$. Las conclusiones son desalentadoras. De todos los documentos analizados, sólo el Plan Nacional de Acción para la Inclusión Social cuenta con una estructura de participación que permite el trabajo de entidades junto a la Administración Pública. En los Informes Nacional Sociales éstas no intervienen, y los Programas Nacionales de Reformas ni siquiera son elaborados por el MSSSI, sino por la Oficina Económica de la Presidencia del Gobierno.

Pero no sólo la legitimidad en cuanto a la elaboración y aplicación de los documentos está en entredicho. También el contenido resulta de dudosa utilidad a la hora de afrontar el reto de sacar del riesgo de pobreza o exclusión social a I,5 millones de personas. Tanto los PNR como los INS, que hacen referencia concreta a elementos y acciones llevados a cabo o que se realizarán en un futuro, tienen un contenido mínimo en lo que a integración social y pobreza se refiere. $Y$ éste se basa, fundamentalmente, en la inserción laboral de las personas.

Consideramos que todo ello no resulta adecuado, respecto del objetivo de pobreza e inclusión social, principalmente por dos motivos. En primer lugar, porque las reformas laborales y las políticas de empleo de los últimos años no han tenido como objetivo ni como resultado la reducción del número de pobres. Al contrario, han contribuido a multiplicar el número de personas incluidas en la categoría de trabajadores pobres en España. Ello viene a ratificar que el principal marco de trabajo para luchar contra la pobreza y la exclusión social (según se desprende de los documentos españoles), es ineficaz. En segundo lugar, no sólo se ha puesto el foco sobre las políticas de empleo por encima de otros elementos como prestaciones y servicios públicos, sino que el recorte en estos dos últimos ha sido sistemático en los últimos años. Una política, en este sentido, acorde a lo que las instituciones europeas han propuesto por medio de sus recomendaciones específicas.

\section{Glosario de siglas}

BCE: Banco Central Europeo

CE: Comisión Europea

CPS: Comité de Protección Social

EAPN: European Anti Poverty Network

INS: Informe Nacional Social

MAC: Método Abierto de Coordinación

MC: Método Comunitario

MSSSI: Ministerio de Sanidad, Servicios Sociales e Igualdad

PDM Procedimiento de Desequilibrios Macroeconómicos.

PAN: Plan de Acción Nacional

PNAIN: Plan Nacional de Acción para la Inclusión Social 2013-2016

PNR: Programa Nacional de Reformas

RSC: Responsabilidad Social Corporativa

SE: Semestre Europeo

\footnotetext{
${ }^{46}$ Somos conscientes de que para analizar de forma completa el grado de participación de estas entidades en la elaboración y aplicación de los documentos debe contarse con la información proporcionada por las mismas. Sin embargo, del análisis de los informes y programas y de las entrevistas realizadas con el MSSSI pueden extraerse conclusiones importantes, sobre todo relativas a la (poca) efectividad de las medidas propuestas respecto del objetivo de pobreza e inclusión social.
} 


\section{Bibliografía}

A Constitution of Democratic Experimentalism. (n.d.).

Atienza, M. (20I3). Curso de argumentación jurídica. Madrid: Trotta.

Borrás, S., \& Radaelli, C. M. (20II). The politics of governance architectures: creation, change and effects of the EU Lisbon Strategy. Journal of European Public Policy, 18(4), 463-484.

Cerrillo i Martínez, A. (2005). La Gobernanza hoy: 10 textos de referencia. Madrid : Instituto Nacional de Administración Pública.

Copeland, P., Mary, Q., \& Daly, M. (20I4). Poverty and social policy in Europe 2020: ungovernable and ungoverned, 42(3), 35I-366.

CPS. (20II). Draft background paper to the SPC from the Ad-Hoc Group on Reinvigorating the Social OMC in the Context of the Europe 2020 Strategy.

EAPN. (20I2). An EU Worth Defending - Beyond Austerity to Social Investment and Inclusive Growth EAPN analysis of the 2012 National Reform Programmes (NRPs ) and National Social Reports (NSRs ), 0032(July), I-96.

Estévez Araujo, J. A. (2008). Que no te den gobernanza por democracia. Mientras Tanto, (108-109), 33-49.

Estévez Araujo, J. A. (20I5). La creación y aplicación del derecho en la gobernanza: el caso del Open Method of Coordination (omc). Sociologia del diritto: Rivista quadrimestrale fondata da Renato Treves. Franco Angeli.

Estévez Araujo, J. A., \& Messina, G. (Eds.). (20I5). La democracia en bancarrota. Madrid: Trotta.

Gérard, P., Ost, F., \& Kerchove, M. van de. (1996). Droit négocié, droit imposé? Bruxelles : Facultés universitaires Saint-Louis.

Habermas, J. (1994). Faktizität und Geltung: Beiträge zur Diskurstheorie des Rechts und des demokratischen Rechtsstaats. : Suhrkamp.

Habermas, J. (1998). Facticidad y validez : sobre el derecho y el estado democrático de derecho en términos de teoría del discurso. Madrid: Trotta.

Horváth, A. (2009). The Social Protection Comittete After the Enlargement of the EU: Discursive Mechanisms of Organisational and Policy Adjustment and Contestation. Central European University.

INS. (20I2). Informe Nacional Social del Reino de España 2012.

INS. (20I4). Informe Nacional Social Del Reino De España 2014.

Jessoula, M. (2015). Europe 2020 and the Fight against Poverty - Beyond Competence Clash, Towards "Hybrid" Governance Solutions? Social Policy \& Administration, 49(4), 490-5II.

Mercado, P. (20I2). DE REGULACIÓN Y LEGITIMACIÓN DEL DERECHO Democratic Experimentalism , New Forms of Regulation and Legitimation of Law, 46, 37-68.

Messina, G. (20I2). Diritto liquido? La governance come nuovo paradigma della politica e del diritto (Franco Ang).

Messina, G., \& Quiroz Vitale, M. A. (20I5). Introduzione: il diritto nuovo. Sociologia del diritto: Rivista quadrimestrale fondata da Renato Treves. Franco Angeli.

PNAIN. (20I3). Plan Nacional de Acción para la Inclusión Social del Reino de España 20132016.

PNR. (20I5). Programa Nacional de Reformas de España 2015.

Pogge, T. W. M. (20I2). \{uOOBF\}Estamos violando los derechos humanos de los pobres del mundo? Cànoves i Samalús: Proteus.

Poiares Maduro, M. (2003). Las Formas del Poder Constitucional de la Unión Europea. Revista de Estudios Políticos, (I 19). 
Ramos Toledano, J. (2016). Bilateralidad, pérdida de soberanía y secretismo. Mientras Tanto, (146).

Rosenau, J. N., \& Czempiel, E. O. (1992). Governance without government: order and change in world politics. Cambridge [etc.] : Cambridge University Press.

Sabel, C. F. (2002). Révolution tranquille de la gouvernance démocratique: vers une démocratie expérimentale. In OCED 2002.

Santos, B. de S., \& Rodríguez Garavito, C. A. (2007). El Derecho y la globalización desde abajo : hacia una legalidad cosmopolita. Rubí : Universidad Autónoma Metropolitana, Cuajimalpa.

Sorensen, E., \& Torfing, J. (2006). Theories of democratic network governance. Basingstoke : Palgrave Macmillan.

Torfing, J. (20I2). Interactive governance advancing the paradigm. Oxford [etc.] : Oxford University Press.

Weiss, J. (20I4). HBR Guide to Negotiating (HBR Guide Series). Harvard Business Review.

Zeitlin, J. (20I4). Experimentalist Governance in European Union. Oxford University Press.

Zeitlin, J., \& Sabel, C. F. (2010). Experimentalist governance in the European Union: towards a new architecture. Oxford : Oxford University Press,. 